\title{
Adesão ao tratamento para Tuberculose Multidroga Resistente (TBMDR): estudo de caso em ambulatório de referência, Niterói (RJ), Brasil
}

\author{
Adherence to Multidrug-Resistant Tuberculosis (MDR-TB) \\ Treatment: case study in a reference outpatient clinic, \\ Niterói (RJ), Brazil
}

\author{
Patricia Valéria Costa ${ }^{1}$ (D), Monica Tereza Christa Machado², \\ Luísa Gonçalves Dutra de Oliveira ${ }^{2}$
}

\begin{abstract}
Resumo
Introdução: Adesão ao tratamento e aumento das taxas de cura resultam da interação de fatores relacionados aos serviços de saúde, pacientes, sociedade e gestão pública. Objetivo: Conhecer os fatores relativos à adesão ao tratamento da tuberculose multidroga resistente (TBMDR), na perspectiva dos usuários, profissionais e gestores. Método: Estudo de caso de abordagem qualiquantitativa, diferentes fontes de evidência, estatística descritiva e análise de conteúdo das categorias: adesão, acesso, acolhimento, vínculo e responsabilização. Resultados: Oferta gratuita da medicação, realização de exames no ambulatório, flexibilidade na agenda, busca de faltosos e vínculo com a equipe de saúde foram descritos como favoráveis à adesão; sendo a dificuldade de acesso aos benefícios sociais e equipe de saúde incompleta como desfavoráveis. Conhecimento sobre a doença atual, o tratamento e o desejo de cura foram relatados como importantes fatores para superar barreiras encontradas para adesão. Conclusão: Apesar da existência de fatores desfavoráveis à adesão, as ações de acolhimento, vínculo e responsabilização desenvolvidas pelo serviço foram suficientes para promover a adesão neste grupo. Recomenda-se a melhoria do acesso aos benefícios assistenciais, implementação de novas formas de comunicação e articulação com outros segmentos públicos e da sociedade para o enfrentamento da TBMDR.
\end{abstract}

Palavras-chave: adesão à medicação; tuberculose; assistência centrada no paciente.

${ }^{1}$ Núcleo de Vigilância Hospitalar, Instituto Estadual de Doenças do Tórax Ary Parreiras, Secretaria de Estado de Saúde do Rio de Janeiro (SES-RJ) - Niterói (RJ), Brasil.

2Departamento de Planejamento em Saúde, Instituto de Saúde da Comunidade, Universidade Federal Fluminense (UFF) - Niterói (RJ), Brasil.

Trabalho realizado no Ambulatório Margareth Pretti Dalcomo, Instituto Estadual de Doenças do Torax Ary Parreiras, Secretaria de Estado de Saúde do Rio de Janeiro (SES/RJ) - Niterói (RJ), Brasil.

Endereço para correspondência: Patricia Valéria Costa - Núcleo de Vigilância Hospitalar, Instituto Estadual de Doenças do Tórax Ary Parreiras, Secretaria de Estado de Saúde do Rio de Janeiro (SES-RJ), Rua Luiz Palmier, 762 - Barreto - CEP: 24110-310 - Niterói (RJ), Brasil - Email: pvcosta.rj@gmail.com

Fonte de financiamento: nenhuma.

Conflito de interesses: nada a declarar. 


\begin{abstract}
Background: Adherence to treatment and high cure rates result from the interaction health services, patients, society and public management. Objective: To determine the factors associated with adherence to multidrug-resistant tuberculosis (MDR-TB) treatment from the perspective of patients, professionals and managers. Method: Case study using quali-quantitative approach, different sources of evidence, descriptive statistics and content analysis based on the categories: adherence, access, embracement, bonding and accountability. Results: The free supply of medicines and exams in the clinic, flexibility of scheduling, active search for no-show patients and relationship with the health team were described as important factors for adherence. Difficult access to social benefits and absence of some professionals of health team were unfavorable factors for adherence. Knowledge of the current illness and its treatment associated with the patient's will were important aspects to overcome the barriers when conducting treatment. Conclusion: Despite the existence of unfavorable factors, the actions of embracement, bonding and accountability were sufficient to promote adherence in this group. It is recommended to further promote access to welfare benefits, new forms of communication and collaboration with other public sectors and society to confront the MDR-TB.
\end{abstract}

Keywords: medication adherence; tuberculosis; patient-centered care.

\section{INTRODUÇÃO}

A multirresistência (MDR), resistência a pelo menos rifampicina e isoniazida, fármacos com importante ação bactericida sobre o bacilo da tuberculose (TB), é hoje um desafio para o controle da doença ${ }^{1}$. No Brasil, houve aumento de $83,4 \%$ dos casos de TBMDR notificados entre 2005 e 2012 e redução de casos de TB sensível no mesmo período. Apesar deste aumento, os casos de TBMDR representavam menos de $1 \%$ do total dos casos de TB notificados naquele período ${ }^{2}$. De 2006 a 2010, os desfechos de tratamento de TBMDR no Brasil apresentaram: redução da taxa de cura no primeiro ano e posterior aumento, de 44\% (2007) para 64\% (2010); manutenção das taxas de óbito e de abandono em torno de $10 \%$ e $14 \%$, respectivamente; e redução do percentual de falência do tratamento a partir de $2009^{2}$. Em 2016, a proporção de TBMDR entre os casos novos atingiu 1,5\% e a taxa de cura teve redução para 52\% em $2014^{3}$.

Diretrizes da Organização Mundial de Saúde (OMS) para o tratamento da TBMDR ratificam a importância de: qualidade do diagnóstico, adequada terapêutica, acesso gratuito à medicação e realização de exames de diagnóstico e acompanhamento. O documento destaca também o contexto social dos pacientes, que barreiras sociais e financeiras ao acesso e à adesão ao tratamento estão presentes, e a importância de assegurar o direito aos benefícios de proteção social, principalmente relacionados ao transporte e à perda de renda ${ }^{4}$.

A longa duração dos esquemas terapêuticos para TBMDR, a carga diária de medicamentos e os efeitos adversos, associados às barreiras sociais e financeiras, podem comprometer a adesão. A OMS recomenda a tomada da medicação supervisionada para garantir seu uso regular e possibilitar a identificação de vulnerabilidades dos pacientes, evitar a ampliação da resistência e aumentar a probabilidade de cura ${ }^{4}$. Outros fatores podem influenciar a adesão ao tratamento: individuais (idade, comorbidades, nível de informação, atitudes e expectativas relativas à doença, ao tratamento e ao sistema de saúde); econômicos (acesso aos benefícios para suportar os custos associados ao tratamento); relacionados ao sistema de saúde (mecanismos que favoreçam a adesão, tais como acesso, motivação e supervisão do tratamento); e sociais (estigma e discriminação) ${ }^{4,5}$. Munro et al. ${ }^{6}$ apontam esses mesmos fatores e incluem o risco de não adesão devido ao uso de álcool e outras drogas, falta de apoio familiar e comunitário. $\mathrm{O}$ abandono de tratamento anterior também é destacado como fator de não adesão ${ }^{7,8}$.

O Programa Nacional de Controle da Tuberculose (PNCT) do Brasil preconiza ações de prevenção e vigilância epidemiológica, diagnóstico precoce e tratamento adequado, além de estratégias operacionais para o Tratamento Diretamente Observado (TDO) e organização dos serviços de saúde, tanto para os casos de TB sensível como para os de resistência9. O Plano Nacional pelo fim da Tuberculose (2017) define a prevenção e o cuidado centrado no paciente como um dos pilares, tendo entre as ações a intensificação da descentralização do diagnóstico e acompanhamento da tuberculose para a Atenção Básica e a implantação da Rede de Teste Rápido Molecular ${ }^{10}$.

O tratamento para TBMDR tem duração de 18 a 24 meses, de acordo com a evolução clínica, laboratorial e radiológica, faixa de peso e presença de comorbidades, dividido em 3 fases, duas intensivas com duração de 2 e 4 meses, respectivamente, e uma de manutenção com 12 meses. Nas duas primeiras, utiliza-se medicação oral e injetável, o que pode dificultar a adesão ao tratamento e a organização da rotina dos serviços. O PNCT recomenda identificação precoce dos efeitos adversos dos fármacos e seu manejo clínico, para evitar interrupção e/ou possível abandono e reduzir complicações e mortalidade ${ }^{11}$.

O acompanhamento dos pacientes TBMDR deve ser mensal em unidades de referência terciária e, em seguida, por um prazo de 5 anos, quadrimestral nos 2 primeiros anos e semestral pelos 3 anos seguintes, com o objetivo de detectar precocemente a recidiva. As unidades de Atenção Básica devem definir a modalidade de TDO de acordo com as diretrizes do Ministério da Saúde (MS) ${ }^{12}$, considerando as melhores estratégias de acordo com as necessidades dos pacientes (frequência, local e responsabilidade pela supervisão) ${ }^{11}$. 
Para melhorar as condições de acesso e acompanhamento dos casos, o Programa de Controle da Tuberculose da SES/RJ (PCT/SES/RJ) redefiniu o fluxo de atendimento em 2011, com adaptações para a realidade de cada região e município. As referências secundárias passaram a acompanhar casos mais complexos, já os de alta complexidade e MDR são tratados pelas 5 unidades de referência terciária do $\mathrm{RJ}^{13}$.

De um modo geral, existe no Brasil e no mundo carência de pesquisas que abordem adesão ao tratamento da TBMDR; estes pacientes, na maioria, passaram por tratamentos anteriores $\mathrm{e}$ se deparam com um esquema terapêutico mais complexo, mais efeitos adversos, duração prolongada e acompanhamento em unidade de referência não raro distante de sua residência.

Este estudo visa identificar os fatores relacionados à adesão ao tratamento por pacientes atendidos em Unidade Ambulatorial de Referência Terciária (UART) cujo acompanhamento é compartilhado com os municípios de residência dos pacientes. Buscou-se: descrição da estrutura, funcionamento e perfil de atendimento da UART; identificação dos desfechos dos casos; conhecimento do perfil socioeconômico dos pacientes; relato dos pacientes e dos profissionais da UART sobre fatores que favorecem ou dificultam a adesão e dinâmica adotada pelos municípios para o acompanhamento dos casos.

\section{MÉTODO}

Estudo de caso único com abordagem qualiquantitativa, sendo o caso selecionado uma UART localizada no Instituto Estadual de Doenças do Tórax Ary Parreiras (IEDTAP) em Niterói, RJ.

Foram utilizados dados primários e secundários, obtidos a partir de entrevistas com roteiro semiestruturado, observação direta, análise de registros e documentos. Para a descrição da estrutura e do funcionamento da UART, realizou-se observação direta com roteiro próprio. Os critérios de inclusão de pacientes para entrevista foram: estarem em tratamento para TBMDR pulmonar por 30 dias ou mais no período da coleta de dados (agosto a outubro de 2014), independentemente da história prévia de abandono, e sem coinfecção TB/HIV. Os 8 profissionais técnicos e administrativos lotados na UART há no mínimo 6 meses, a diretora do IEDTAP e 3 coordenadores do PCT de municípios da área de cobertura também foram entrevistados.

O Sistema de Informação de Tratamentos Especiais da Tuberculose (SITETB) forneceu informações da unidade estudada e do acompanhamento individual dos casos selecionados.

A observação direta de 4 reuniões com PCT/SES, UART e PCT Municipais contribuiu para a compreensão do fluxo de atendimento, da responsabilização e das dificuldades encontradas pelas equipes quanto ao acompanhamento dos casos.

A análise descritiva dos dados quantitativos mostrou o perfil dos participantes da pesquisa e a caracterização da UART, e a abordagem qualitativa teve como base a análise de conteúdo ${ }^{14}$.
A adesão foi a categoria de análise para identificação das unidades de registro nas diversas fontes de informação. Outras questões relacionadas a essa categoria foram também analisadas: acesso, acolhimento, vínculo e responsabilização.

Para discutir os fatores que interferem na adesão ao tratamento, foram considerados os termos adherence (adesão), que considera o paciente capaz de decidir e assumir responsabilidades por seu tratamento, com autonomia e habilidade para aceitar ou não as recomendações, e compliance (obediência) que responsabiliza o paciente pelo insucesso do tratamento, quando este não cumpre as ordens médicas ${ }^{15}$.

Este estudo teve anuência da Direção do IEDTAP e do Coordenador Técnico da UART e aprovação pelo Comitê de Ética em Pesquisa do Hospital Universitário Antonio Pedro via Plataforma Brasil (CAAAE3128854.3.0000.5243). Foi garantida livre participação na pesquisa e sigilo das fontes mediante assinatura do Termo de Consentimento Livre e Esclarecido.

\section{RESULTADOS E DISCUSSÃO}

\section{Infraestrutura e funcionamento}

Inaugurado em 2004, o Ambulatório Margareth Pretti Dalcomo é uma das UARTs do RJ para tratamento de casos de TBMDR, tuberculose em situações especiais e micobacterioses atípicas não tuberculosas (MNT), abrangendo os municípios da Região Metropolitana II (RM II), Magé, baixada litorânea e parte da região serrana. Por ser vinculado ao IEDTAP, a unidade contava com exames laboratoriais, radiografias, broncoscopia e assistência farmacêutica, além de profissionais experientes no tratamento da TBMDR. Antes da inauguração do ambulatório, somente o Centro de Referência Professor Hélio Fraga (CRPHF), localizado no município do RJ, acompanhava esses casos. A descentralização da assistência proporcionada pela criação de novas UARTs, segundo áreas de abrangência, foi considerada medida favorável à adesão, por reduzir as distâncias percorridas pelos pacientes e despesas com transporte.

Cabia à SES/RJ a manutenção e custeio da UART, assim como a lotação dos profissionais. Os medicamentos, fornecidos pelo MS através do CRPHF, eram retirados pelo IEDTAP, gerando transtorno devido à ausência de veículo específico para o expediente.

O Ambulatório, localizado na entrada do IEDTAP, era composto por: sanitários para pacientes; consultórios (2); sala de espera; assistência farmacêutica; pré-consulta; e administração. Os espaços fechados de atendimento contavam com filtros HEPA (high efficiency particulate air) e ventilador na sala de espera em fluxo contrário à entrada de ar. Funcionava nos dias úteis das 8 às $17 \mathrm{~h}$ com equipe multiprofissional, mas apenas 4 turnos de atendimento médico em 3 dias da semana.

A coleta de material para exames laboratoriais era realizada nos dias de consulta e os resultados disponibilizados na consulta 
subsequente. Os pacientes eram orientados quanto à coleta de escarro no domicílio. Se necessário, o escarro induzido era realizado no setor de broncoscopia do IEDTAP. O resultado da baciloscopia era disponibilizado imediatamente para a equipe.

$\mathrm{O}$ trajeto íngreme para o setor de radiologia dificultava o acesso de pacientes em condições clínicas precárias. Os demais exames de imagem eram encaminhados para outras unidades, após esgotadas as possibilidades de realização no município de residência. A broncoscopia era feita no IEDTAP mediante agendamento.

A organização da agenda, baseada nos turnos de atendimento médico, visava atender em dias e/ou horários distintos casos de TBMDR, TB extensivamente resistente (XDR) ${ }^{1}$, MNT, pós-alta e outras avaliações, e, ainda, segundo o município de residência. Esta medida visava garantir a biossegurança no atendimento, facilitar o transporte dos pacientes pelos municípios e a retirada de medicação para TDO, conforme fluxograma do PCT Estadual. $\mathrm{O}$ atendimento dos pacientes faltosos e dos não agendados era providenciado, priorizando os com maior risco ou história prévia de abandono.

A vigilância dos casos era intensificada através de planilha semanal com atendimentos realizados, datas das consultas subsequentes e casos faltosos, encaminhada ao PCT Estadual e aos PCTs municipais. Havia reunião bimestral da UART com as Coordenações dos PCTs municipais e estadual para discussão de casos clínicos, enfatizando a responsabilidade das equipes do território no acompanhamento dos casos.

A assistência farmacêutica no próprio ambulatório facilitava o acesso dos pacientes e o gerenciamento dos medicamentos. Após notificação e validação do caso no SITETB, a medicação era dispensada para 4 meses. A UART não tinha acesso à Internet, impossibilitando a notificação imediata. Para não comprometer a gestão dos casos e liberação da medicação, a rede era acessada em outro setor do hospital.

A sala de recepção não era utilizada para ações de educação em saúde, feitas apenas individualmente nas consultas. Havia urna da ouvidoria, mas não quadro com horário de funcionamento e escala de profissionais.

A estrutura física e o funcionamento da UART não representavam barreiras para o acesso ao tratamento, mas comprometiam o fluxo das ações e acarretavam maior tempo de espera. A demora para atendimento associada à forma de organização do serviço era vista pelos usuários como um fator de desmotivação e insatisfação ${ }^{16}$. Estudo no município do Rio de Janeiro mostrou que as dimensões acessibilidade/conveniência, ambiente físico e disponibilidade de recursos nas unidades de saúde com PCT foram as que geraram os menores índices

\footnotetext{
Tuberculose resistente a rifampicina, isoniazida, uma fluoroquinolona e a
} pelo menos um dos medicamentos injetáveis de segunda linha. de satisfação dos usuários ${ }^{17}$. Lima et al. ${ }^{18}$, em estudo sobre abandono de tratamento da TB, apontam que usuários avaliaram positivamente a presteza no agendamento da primeira consulta, na solicitação de exames e dispensação de medicação, e como aspecto negativo a demora para atendimento.

A realização de exames nos locais de atendimento e o acesso à equipe multiprofissional estão associados à adesão ${ }^{17,19}$. No presente estudo, o fácil acesso às consultas, à medicação e aos exames foi citado por pacientes e profissionais como aspecto importante para o tratamento. Os problemas apontados foram: fracionamento na dispensação da medicação, com deslocamento do paciente fora dos dias de consulta, e dificuldade de acesso a medicamentos para tratar efeitos adversos. A garantia de transporte é condição fundamental para realizar o tratamento, principalmente numa população socialmente vulnerável ${ }^{20,21}$.

Segundo dados do SITETB, no período de 2004 a 2014, foram cadastrados na UART 247 pacientes. Destes, 207 eram casos encerrados no período da coleta de dados deste estudo, sendo 168 de MDR. O perfil dos casos de TBMDR encerrados é apresentado na Tabela 1, de acordo com sexo, faixa etária, escolaridade, ocupação, consumo de álcool, tabaco e outras drogas e situação de encerramento.

Os prontuários dos pacientes que abandonaram o tratamento registravam fatores de risco para abandono ou uso irregular da medicação, como consumo de bebida alcoólica, ausência de apoio familiar e inexistência de vínculo formal de trabalho. O número de casos de TBMDR encerrados como abandono ao longo dos 10 anos de atividade da UART aumentou a partir de 2009 e, mais expressivamente, em 2014 (Gráfico 1), totalizando 42 pacientes (25\% de abandono).

Na percepção da equipe de saúde, o aumento do número de pacientes usuários de álcool e outras drogas levou a maior abandono.

De um tempo para cá modificou [...] o perfil do paciente mudou um pouco. Nós temos pacientes usuários de drogas, o que é muito difícil de lidar, [...] abandonam qualquer tipo de tratamento... (Profissional 4).

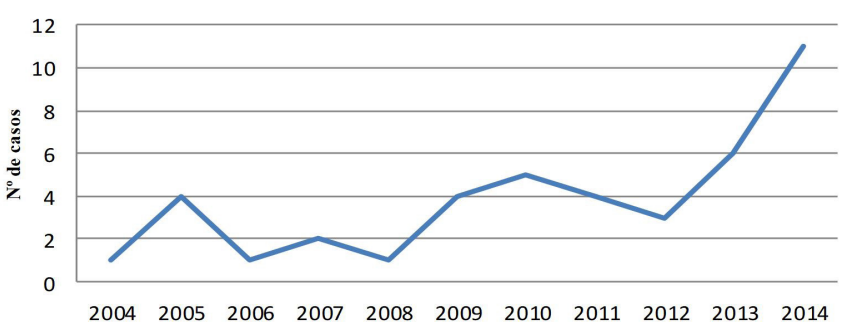

Gráfico 1. Casos de abandono registrados na Unidade Ambulatório Margareth Pretti Dalcomo (Sistema de Informação de Tratamentos Especiais da Tuberculose - SITETB), de acordo com o ano do término do tratamento (2004 a 2014) 
Tabela 1. Perfil dos casos de tuberculose multirresistente (TBMDR) encerrados no Ambulatório Margareth Pretti Dalcomo, Niterói - RJ, 2004-2014

\begin{tabular}{|c|c|c|c|}
\hline \multicolumn{2}{|c|}{ Características } & \multirow{2}{*}{$\begin{array}{c}\mathbf{N}^{\mathbf{0}} \\
5\end{array}$} & \multirow{2}{*}{$\begin{array}{c}\% \\
28\end{array}$} \\
\hline \multirow{3}{*}{ Sexo } & Feminino & & \\
\hline & Masculino & 13 & 72 \\
\hline & 18 a 24 anos & 2 & 11 \\
\hline \multirow[t]{3}{*}{ Faixa etária } & 25 a 59 anos & 10 & 56 \\
\hline & $>60$ anos & 6 & 33 \\
\hline & Ensino Fundamental ( $1^{\circ}$ segmento) & 5 & 28 \\
\hline \multirow{3}{*}{ Escolaridade } & Ensino Fundamental ( $2^{\circ}$ segmento $)$ & 9 & 50 \\
\hline & Ensino médio & 3 & 17 \\
\hline & Ensino superior & 1 & 5 \\
\hline \multirow{5}{*}{ Ocupação } & Do lar & 4 & 22 \\
\hline & Com vínculo formal de trabalho & 3 & 17 \\
\hline & Sem vínculo formal de trabalho & 10 & 56 \\
\hline & Aposentado & 1 & 5 \\
\hline & Álcool & 9 & 50 \\
\hline \multirow[t]{2}{*}{ Relato do consumo de substâncias } & Tabaco & 4 & 22 \\
\hline & Outras drogas & 2 & 11 \\
\hline \multirow{8}{*}{ Situação de encerramento } & Abandono & 42 & 25 \\
\hline & Cura & 51 & 30 \\
\hline & Falência do tratamento & 10 & 6 \\
\hline & Mudança de diagnóstico & 1 & 0,5 \\
\hline & Mudança de esquema & 1 & 0,5 \\
\hline & Óbito & 13 & 8 \\
\hline & Óbito por outras causas & 5 & 3 \\
\hline & Tratamento completo & 45 & 27 \\
\hline
\end{tabular}

Fonte: Sistema de Informação de Tratamentos Especiais da Tuberculose (SITETB)

\section{Percepção dos usuários}

Durante a coleta de dados, havia 25 pacientes com TBMDR em tratamento. Os 18 pacientes selecionados, 5 mulheres e 13 homens, com idade média de 47 anos, eram residentes nos municípios de Niterói (5), São Gonçalo (8), Cachoeira de Macacu (1), Araruama (1), Itaboraí (2) e Magé (1), todos com resistência adquirida. Observou-se predominância de baixa escolaridade (78\% tinham até ensino fundamental incompleto) e ausência de renda fixa em $78 \%$ dos pacientes. Metade deles relatou contar com apoio familiar. O mesmo número informou consumo de bebida alcoólica; já o tabaco e outras drogas eram pouco comuns. As características socioeconômicas dos pacientes deste estudo são similares às encontradas na literatura ${ }^{22-25}$. A condição clínica inviabilizava, por vezes, a atividade laborativa, comprometendo o orçamento familiar e agravando ainda mais a situação econômica. Apesar de a equipe acionar os dispositivos de amparo social, nenhum dos pacientes, no momento da entrevista, declarou receber qualquer dos benefícios existentes. Relataram dificuldades nos trâmites administrativos das instâncias responsáveis pela concessão dos benefícios e frequentes deslocamentos sem resolutividade. Alguns estudos também ressaltam a dificuldade e irregularidade em acessar os benefícios com base nos critérios definidos ${ }^{16,21-23}$. O passe livre para transporte público era frequentemente obtido, porém muitas vezes em quantidade insuficiente. Esse problema, descrito em outros estudos ${ }^{20,26}$, pode comprometer o orçamento para as necessidades básicas. A descontinuidade dos benefícios e a frustração causada pelo não recebimento deles são fatores desestimulantes para muitos pacientes. $\mathrm{O}$ fortalecimento da articulação intra e intersetorial para garantia dos direitos humanos e da cidadania nas ações de controle da doença é uma das estratégias políticas de apoio previstas em documento recente do $\mathrm{MS}^{10}$.

A experiência de tratamento anterior pode influenciar o comportamento do paciente em novo episódio da doença, seja na percepção dos sinais e sintomas, na busca da assistência ou no cuidado em relação ao tratamento. Todos os entrevistados relataram um ou mais tratamentos anteriores e 4 relacionaram os abandonos anteriores ao uso de drogas, álcool e outras práticas, além da falta de apoio familiar. O diagnóstico de TBMDR ocorreu para alguns após evolução clínica desfavorável no tratamento para TB sensível; outros tiveram esse diagnóstico no segundo episódio da doença, após encerramento de tratamento anterior por cura. Embora a literatura aponte fatores preditivos de abandono do tratamento relacionados ao paciente e ao estilo de vida, ao serviço de saúde e à terapêutica ${ }^{27}$, neste estudo, verificamos que a experiência negativa com o resultado anterior 
influenciou a atitude em relação ao novo tratamento, gerando receio de um desfecho desfavorável e consequentemente maior empenho para a obtenção da cura.

Já tratei antes por 2 vezes, mas não conclui o tratamento [...] Nunca pensei em abandonar este [...] pois eu vi o que sofri a primeira vez [...] se eu soubesse como era, eu teria me tratado direito (Usuário 6).

A equipe deve estar atenta para utilizar essa situação como canal de diálogo, estimulando a adesão. Os pacientes entrevistados desconheciam anteriormente as formas graves da $\mathrm{TB}$, visto que em geral somente as formas mais comuns da doença são abordadas pelos profissionais de saúde e meios de comunicação.

Houve relatos de uso irregular da medicação para TBMDR motivado pela depressão, alcoolismo, volume de medicamentos e falta de apoio da equipe de saúde. A ocorrência de efeitos adversos foi relatada por mais da metade dos pacientes, porém a grande maioria referiu que medidas terapêuticas e orientações para manejo dos efeitos foram eficazes, não comprometendo a continuidade do tratamento. Pouco mais da metade dos pacientes realizava o TDO na atenção básica. Em alguns casos, a administração dos medicamentos era feita por familiar ou vizinho. Cruz et al. (2012) ressaltam os ajustes realizados pelos serviços para facilitar o atendimento e a adaptação do usuário ao tratamento ${ }^{28}$.

A medicação injetável foi considerada como dificuldade, tanto por exigir um profissional como pela dor das aplicações. O TDO, embora reconhecido como estratégia de fortalecimento da adesão, apresentava dificuldades estruturais e operacionais. No estudo de Cardoso et al. ${ }^{29}$, os profissionais relataram que a ausência de incentivos (vale transporte e cesta básica) compromete a realização do TDO na unidade de saúde diariamente.

A rotatividade de profissionais e de coordenadores do PCT na RM II acarretava necessidade contínua de articulação e treinamento para as equipes, e comprometia o vínculo e a responsabilização. Em 2011, estudo de avaliação da implantação da estratégia TDO na Paraíba ${ }^{30}$ apontou como fragilidades: resistência dos profissionais em reorganizar a rotina para inserir o TDO, descontinuidade dos coordenadores do PCT e do pessoal técnico, rotatividade dos profissionais da Estratégia de Saúde da Família (ESF) e falta de qualificação profissional. A duração e complexidade do tratamento, presença de efeitos adversos, distância a ser percorrida e ausência de benefícios sociais, a princípio, não foram motivos para abandono de tratamento pelos pacientes deste estudo. Entretanto, há que se considerar que a soma desses fatores ou, ainda, a permanência deles nos diferentes momentos da vida e fases do tratamento podem transformar-se em motivação para abandono e/ou irregularidade no tratamento. A violência, uma realidade comum, foi apontada como obstáculo ao acesso ao tratamento:
[...] eu me tratava [...], mas como tinha esse negócio de faç̧ão eu não pude ficar mais lá, estavam atrás do meu filho. E não pude voltar mais pra lá para continuar o tratamento (Usuário 6).

Paula e Aguiar ${ }^{31}$ descrevem a violência como barreira para a continuidade do tratamento e para o deslocamento das equipes de saúde no território.

No grupo estudado, a doença levou ao isolamento e à pouca participação social por sentirem-se rejeitados:

[...] eu notava que as pessoas quando falavam comigo, recuavam, entendeu? Eu não tenho coragem de ir a lugar nenhum. Eu vou num mercado e já acho que todo mundo tá me olhando [...] (Usuário 1).

Esse resultado foi também observado em outros estudos ${ }^{11,14,22}$.

\section{A visão dos profissionais}

A equipe era composta por 2 pneumologistas, farmacêutico, assistente social, enfermeira, técnico de enfermagem, auxiliar de enfermagem, agente de saúde pública e agente administrativo. A baixa rotatividade de profissionais permitia a formação de um corpo técnico com experiência, porém a maior parte não recebia capacitação permanente em relação ao tratamento clínico e a outros fatores relacionados à adesão. No decorrer deste estudo, a assistente social foi transferida, o que pode ter prejudicado o atendimento. Medeiros et al. ${ }^{32}$ aponta como fatores para a alta rotatividade de profissionais: precarização do vínculo de trabalho, fragmentação da formação, estilo de gestão autoritário, ausência de vínculo com a comunidade e más condições de trabalho. $\mathrm{O}$ estudo de Souza et al. ${ }^{19}$ aponta que o maior percentual de adesão ( $\geq 85 \%$ ) ocorreu nas unidades que possuíam equipe de saúde completa.

Os profissionais da UART, em sua maioria, compreendiam a adesão ao tratamento como resultado do envolvimento da equipe, dos pacientes e dos familiares, e ressaltaram o papel da equipe no esclarecimento do processo de adoecimento, reabilitação e cura. Na sua opinião, a demonstração da vontade do paciente em se tratar se dava pelo uso correto da medicação e mudanças no estilo de vida. Enfatizaram o papel da equipe como apoiadora do processo de adesão, mediante ações educativas (realizadas exclusivamente durante as consultas), oferta de atendimento multiprofissional e pactuação do tratamento. Somente um profissional expressou a ideia de adesão unicamente como obediência ao tratamento prescrito.

Os profissionais consideraram que o uso irregular da medicação decorreu da dificuldade de entendimento, consumo de álcool, incompreensão sobre a gravidade da doença ou melhora dos sintomas após o início do tratamento. Relataram rejeição enfrentada por alguns pacientes dentro do núcleo familiar e/ou social, adotando por vezes medidas excessivas para evitar o contágio, mesmo após orientações da equipe. A recusa de alguns em serem tratados próximos à sua residência 
mostrou que não queriam ser reconhecidos como portadores da doença. A fragilidade ou rompimento dos laços familiares entre os pacientes usuários de álcool e outras drogas dificultou contato com as famílias nas situações de abandono. A equipe deve identificar essas dificuldades, muitas vezes omitidas ou não verbalizadas claramente. Em um estudo de revisão integrativa sobre fatores associados ao abandono do tratamento da tuberculose, Chirinos e Meirelles ${ }^{33}$ mostraram a associação do consumo diário de álcool com o abandono.

A articulação intra e intersetorial foi considerada importante para favorecer a adesão, com políticas públicas de garantia e ampliação dos direitos sociais e das ações de combate à dependência química. A necessidade de equipe multidisciplinar foi reconhecida pelos entrevistados, destacando-se a importância do psicólogo, que deixou de compor a equipe por questões orçamentárias. Esse profissional atuava no suporte aos pacientes psiquiátricos e dependentes químicos, favorecendo a adesão ao tratamento, e no apoio à equipe na abordagem desses pacientes.

A UART tinha boa articulação com as Coordenações dos PCTs municipais e conseguia absorver os casos de TBMDR encaminhados; entretanto, existiam dificuldades para acesso a exames e consultas com especialistas e internação hospitalar na própria unidade, devido às condições de biossegurança. A dificuldade em acessar serviços mais complexos é observada não só no tratamento da tuberculose ${ }^{34,35}$.

As estratégias de pactuação, de acolhimento e vínculo desenvolvidas pela equipe diante dos fatores individuais e os relacionados ao tratamento se aproximavam mais do conceito de adesão na perspectiva do termo adherence; entretanto, são necessárias capacitação permanente e ampliação da discussão com a equipe e gestores para viabilizar ações que favoreçam a adesão. A baixa rotatividade dos profissionais contribuía para a formação do vínculo, proporcionando um canal de diálogo e confiança durante os atendimentos individuais. Contudo, a atenção à saúde para além das questões relacionadas à medicação e exames encontrava-se comprometida devido à ausência de alguns profissionais e da não realização de ações coletivas de educação em saúde, sendo um desafio para a integralidade da atenção.

\section{CONSIDERAÇÕES FINAIS}

Compreendida como resultado de uma rede complexa de fatores que envolvem serviços de saúde, pacientes, sociedade e gestão pública, a adesão ao tratamento representa um desafio para alcance das metas de cura para a tuberculose.

Neste estudo de caso, foram encontrados fatores associados à não adesão relatados na literatura, tanto para a tuberculose sensível como para a resistente. Entretanto, foram identificados como fatores favoráveis à adesão a experiência negativa com tratamento anterior e a percepção da gravidade da doença, com possibilidade de evolução para a forma de resistência extensiva.

A regularidade na oferta da medicação específica, a realização de exames na própria unidade e a flexibilidade da agenda foram fatores positivos para a adesão, bem como a presença e vínculo com a equipe multiprofissional.

A dificuldade na obtenção dos benefícios de Prestação Continuada, transporte e alimentação foram barreiras enfrentadas pelos pacientes, que necessitavam de apoio familiar ou outros mecanismos para superá-las.

É urgente a adoção de estratégias que favoreçam a adesão ao tratamento para enfrentar o aumento de casos de resistência aos fármacos, evitando o aumento dos custos para o setor saúde.

\section{REFERÊNCIAS}

1. Arbex MA, Varella MCL, Siqueira HR, Mello FAF. Drogas antituberculose: Interações medicamentosas, efeitos adversos e utilização em situações especiais. Parte 1: fármacos de primeira linha. J Bras Pneumol. 2010;36(5):626-40. http://dx.doi.org/10.1590/S1806-37132010000500016. PMid:21085830.

2. World Health Organization [Internet]. MDRTB indicators. 2014 [citado em 2014 jan 16]. Disponível em: http: who.int/tb/data

3. World Health Organization. Global tuberculosis report 2017. Geneva: WHO; 2017.

4. World Health Organization. Companion handbook to the WHO guidelines for the programmatic management of drug-resistant tuberculosis. Geneva: WHO; 2014.

5. Rabahi MF, Silva JLR Jr, Ferreira ACG, Tannus-Silva DGS, Conde MB. Tratamento da tuberculose. J Bras Pneumol. 2017;43(5):472-86. http:// dx.doi.org/10.1590/s1806-37562016000000388. PMid:29340497.

6. Munro SA, Lewin SA, Smith HJ, Engel ME, Fretheim A, Volmink J. Patient adherence to Tuberculosis Treatment: A Systematic Review of Qualitative
Research. PLoS Med. 2007;4(7):1230-45. http://dx.doi.org/10.1371/journal. pmed.0040238. PMid:17676945.

7. Silva CCAV, Andrade MS, Cardoso MD. Fatores associados ao abandono do tratamento de tuberculose em indivíduos acompanhados em unidades de saúde de referência na cidade do Recife, Estado de Pernambuco, Brasil, entre 2005 e 2010. Rev Epidemiol Saúde. 2013;22(1):77-85.

8. Silva PF, Moura GS, Caldas AJM. Fatores associados ao abandono de tratamento de tuberculose pulmonar no Maranhão, Brasil, no período de 2001 a 2010. Cad Saude Publica. 2014;30(8):1745-54. http://dx.doi. org/10.1590/0102-311X00124513. PMid:25210913.

9. Brasil. Ministério da Saúde. Secretaria de Vigilância em Saúde. Departamento de Vigilância Epidemiológica. Manual de recomendações para o controle da tuberculose no Brasil. Brasília; 2011. (Série A. Normas e Manuais Técnicos).

10. Brasil. Ministério da Saúde. Secretaria de Vigilância em Saúde. Departamento de Vigilância das Doenças Transmissíveis. Brasil livre da tuberculose: plano 
nacional pelo fim da tuberculose como problema de saúde pública. Brasília; 2017.

11. Brasil. Ministério da Saúde. Secretaria de Vigilância em Saúde. Departamento de Vigilância Epidemiológica. Resitência aos fármacos antituberculose. In: Brasil. Ministério da Saúde. Manual de recomendações para o controle da tuberculose no Brasil. Brasília; 2011. (Série A. Normas e Manuais Técnicos).

12. Brasil. Ministério da Saúde. Secretaria de Vigilância em Saúde. Departamento de Vigilância Epidemiológica. Tratamento Diretamente Observado (TDO) da tuberculose na atenção básica: protocolo de enfermagem. Brasília; 2011.

13. Rio de Janeiro. Secretaria de Estado de Saúde. Gerência de Pneumologia Sanitária. Rotina para atendimento de tuberculose resistente às drogas [Internet]. 2011 [citado em 2014 jan 16]. Disponível em: www.riocomsaude. rj.gov.br

14. Bardin L. Análise de conteúdo. Lisboa: Edições 70; 2008.

15. Queiroz R, Nogueira PA. Diferenças na adesão ao tratamento da Tuberculose em relação ao sexo no Distrito de Saúde da Freguesia do Ó/Brasilândia - São Paulo. Saude Soc. 2010;19(3):627-37. http://dx.doi.org/10.1590/ S0104-12902010000300014.

16. Lafaiete RS, Silva CB, Oliveira MG, Motta MCS, Villa TCS. Investigação sobre o acesso ao tratamento de tuberculose em Itaboraí/RJ. Esc Anna Nery. 2011;15(1):47-53. http://dx.doi.org/10.1590/S1414-81452011000100007.

17. Portela MC, Lima SML, Brito C, Ferreira VMB, Escosteguy CC, Vasconcellos MTL. Programa de Controle da tuberculose e satisfação dos usuários, Rio de Janeiro. Rev Saude Publica. 2014;48(3):497-507. http://dx.doi.org/10.1590/ S0034-8910.2014048004793. PMid:25119945.

18. Lima MB, Mello DA, Morais APP, Silva WC. Estudo de casos sobre abandono do tratamento da tuberculose: avaliação do atendimento, percepção e conhecimentos sobre a doença na perspectiva dos clientes (Fortaleza, Ceará, Brasil). Cad Saude Publica. 2001;17(4):877-85. http://dx.doi.org/10.1590/ S0102-311X2001000400021. PMid:11514868.

19. Souza MSPL, Pereira SM, Marinho JM, Barreto ML. Características dos serviços de saúde associadas à adesão ao tratamento da tuberculose. Rev Saude Publica. 2009;43(6):997-1005. http://dx.doi.org/10.1590/S003489102009005000085. PMid:20027499.

20. Palha PF, Silva LMC, Wysocki AD, Andrade RLP, Protti ST, Scatena LM, et al. Acesso aos serviços de atenção à tuberculose: análise da satisfação dos doentes. Rev Esc Enferm USP. 2012;46(2):342-8. http://dx.doi.org/10.1590/ S0080-62342012000200011. PMid:22576537.

21. Mazzei AMA, Monroe AA, Sassaki CM, Gonzales RIC, Villa TCS. Suporte social para portador de tuberculose no serviço de saúde e na comunidade. Bol. Pneumol. Sanit. 2003;11(2):41-6.

22. Vieira AA, Ribeiro SA. Adesão ao tratamento da tuberculose após a instituição da estratégia de tratamento supervisionado no município de Carapicuíba, Grande São Paulo. J Bras Pneumol. 2011;37(2):223-31. http:// dx.doi.org/10.1590/S1806-37132011000200013. PMid:21537659.

23. Sripad A, Castedo J, Danford N, Zaha R, Freile C. Effects of Ecuador's national monetary incentive program on adherence to treatment for drugresistant tuberculosis. Int J Tuberc Lung Dis. 2014;18(1):44-8. http://dx.doi. org/10.5588/ijtld.13.0253. PMid:24365551.
24. Costa PV, Figueiredo SP, Oliveira LGD, Senna AL, Siqueira RCAG. Demora no diagnóstico da tuberculose pulmonar em 5 municípios da região metropolitana do RJ, Brasil, 2009-2010. Cad Saude Colet. 2012;20(2):195202.

25. Braga JU, Pinheiro JS, Matsuda JS, Barreto JAPB, Feijão AMM. Fatores associados ao abandono do tratamento da tuberculose nos serviços de atenção básica em dois municípios brasileiros, Manaus e Fortaleza, 2006 a 2008. Cad Saude Colet. 2012;20(2):225-33.

26. Villa TCS, Andrade RLP, Arakawa T, Magnabosco GT, Beraldo AA, Monroe AA, et al. Satisfação do usuário como os serviços de atenção à tuberculose em Ribeirão Preto, 2008. Cad Saude Colet. 2012;20(2):234-43.

27. Portela NLC. Fatores associados ao abandono do tratamento da tuberculose: uma revisão integrativa da literatura. Revista Univap. 2015;21(38):15-25. http://dx.doi.org/10.18066/revistaunivap.v21i38.321.

28. Cruz MM, Cardoso GCP, Abreu DMF, Decotelli PV, Chrispim PPM, Borestein JS, Santos EM. Adesão ao tratamento diretamente observado da tuberculose. Cad Saude Colet. 2012;20(2):217-24.

29. Cardoso GCP, Cruz MM, Abreu DMF, Decotelli PV, Chrispim PPM, Borestein JS, et al. A conformidade das ações do tratamento diretamente observado para tuberculose na perspectiva dos profissionais de duas unidades de saúde da cidade do Rio de Janeiro. Cad Saude Colet. 2012;20(2):203-10.

30. Sá LD, Andrade MN, Nogueira JA, Villa TCS, Figueiredo TMRM, Queiroga RPF, et al. Implantação da estratégia DOTS no controle da tuberculose na Paraíba: entre o compromisso político e o envolvimento das equipes do programa de saúde da família (1999-2004). Cien Saude Colet. 2011;16(9):391724. http://dx.doi.org/10.1590/S1413-81232011001000028. PMid:21987335.

31. Paula HC, Aguiar AC. Abandono de tratamento da tuberculose na Estratégia de Saúde da Família: estudo qualitativo em uma área programática do Rio de Janeiro. Rev Baiana de Saúde Pública. 2013;37(1):192-204. http://dx.doi. org/10.22278/2318-2660.2013.v37.n1.a356.

32. Medeiros CRG, Junqueira AG, Schwingel G, Carreno I, Jungles LA, Saldanha OM. A rotatividade de enfermeiros e médicos: um impasse na implementação da Estratégia de Saúde da Família. Cien Saude Colet. 2010;15(Supl 1):152131. http://dx.doi.org/10.1590/S1413-81232010000700064. PMid:20640314.

33. Chirinos NEC, Meirelles BHS. Fatores associados ao abandono de tratamento da Tuberculose: uma visão integrativa. Texto Contexto Enferm. 2011;20(3):399-406. http://dx.doi.org/10.1590/S0104-07072011000300023.

34. Mascarenhas MTM. Avaliando a implementação da atenção básica em saúde no município de Niterói, RJ: estudos de caso em unidade básica de saúde e módulo do programa médico de família [tese]. Rio de Janeiro: Escola Nacional de Saúde Pública Sergio Arouca, Fundação Oswaldo Cruz; 2003.

35. Oliveira LGD, Natal S. Avaliação de implantação do Programa de Controle da Tuberculose e sua integração com o Programa Médico de Família: estudo de caso em Niterói (RJ). In: Santos EM, Cruz MM, editores. Avaliação em saúde: dos modelos teóricos à prática da avaliação de programas de controle de processos endêmicos. Rio de Janeiro: FIOCRUZ; 2014. p. 161-79.

Recebido em: Ago. 18, 2017 Aprovado em: Dez. 13, 2018 